\title{
A comparison of five extraction methods for extracellular polymeric substances (EPS) from biofilm by using three- dimensional excitation-emission matrix (3DEEM) fluorescence spectroscopy
}

\author{
Xiangliang Pan ${ }^{1 *}$, Jing Liu' ${ }^{2}$, Daoyong Zhang ${ }^{2 *}$ Xi Chen ${ }^{1}$, Lanhai Li', Wenjuan Song ${ }^{1}$, Jianying Yang ${ }^{1}$ \\ ${ }^{1}$ Key Laboratory of Biogeography and Bioresource in Arid Land, Xinjiang Institute of Ecology and Geography, \\ Chinese Academy of Sciences, Urumqi, Xinjiang China, 830011 \\ ${ }^{2}$ State Key Laboratory of Environmental Geochemistry, Institute of Geochemistry, Chinese Academy of Sciences, \\ Guiyang, Guizhou, China, 550002
}

\begin{abstract}
Two physical methods (centrifugation and ultrasonication) and 3 chemical methods (extraction with EDTA, extraction with formaldehyde, and extraction with formaldehyde plus $\mathrm{NaOH}$ ) for extraction of EPS from alga-bacteria biofilm were assessed. Pretreatment with ultrasound at low intensity doubled the EPS yield without significant modification of the composition of EPS. Extraction with EDTA or extraction with formaldehyde plus $\mathrm{NaOH}$ increased yield by about 1 order of magnitude compared with other methods. However, the protein and polysaccharide content in EPS prepared with EDTA or formaldehyde plus $\mathrm{NaOH}$ were low. Two fluorescence peaks belonging to protein-like peaks and 2 fluorescence peaks belonging to humic acid-like substances were found in 3DEEM fluorescence spectra of all the EPS samples prepared using different methods. Fulvic-like fluorescence was detected only in the EPS extracted with formaldehyde plus NaOH. Location of, and fluorescence intensity at, each peak were clearly affected by the extraction methods. Dialysis was also found to be an important factor influencing the yield, composition and fluorescence characteristics of EPS.
\end{abstract}

Keywords: biofilm, extracellular polymeric substances, 3DEEM fluorescence spectroscopy

\section{Introduction}

Extracellular polymeric substances (EPS) are secreted by microorganisms with their growth, and accumulate on cell surfaces (Liu and Fang, 2002a; Tsuneda et al., 2003). EPS is composed of some high molecular weight compounds, including polysaccharide, protein, nucleic acids, humic substances, and ionisable functional groups like carboxylic, phosphoric amino and hydroxyl groups (Tsuneda et al., 2003; Guibaud et al., 2008). EPS plays a key role in the formation of biofilm. It makes the biofilm stable and forms a barrier protecting the biofilm from harmful effects, as well as reducing water loss from the cell to environment (Laspidou and Rittmann, 2002). EPS can efficiently remove heavy metals and organic contaminants since EPS has a large number of negatively-charged functional groups (Zhang et al., 2006; Liu et al., 2001; Sheng et al., 2005; Bhaskar and Bhosle, 2006). EPS can form multiple complexes with many heavy metal ions and consequently has a great impact on geochemical behaviour, bioavailability and toxicity of heavy metal ions (Selck et al., 1999). EPS has been considered as one of the most important types of dissolved organic matter (DOM) influencing the fate of heavy metals in the aquatic environment (Bhaskar and Bhosle, 2006). In the past several decades EPS has been extensively studied with regard to its physicochemical properties, interaction with contaminants and role in biofilm formation (Laspidou and

\footnotetext{
* To whom all correspondence should be addressed.

푤 086-851-5890459; fax: 086-851-5890459; e-mail: Ehang-daoyong@163.com; xiangliangpan@163.com Received 11 June 2009; accepted in revised form 27 November 2009.
}

Rittmann, 2002; Bhaskar and Bhosle, 2006; Meisen et al., 2008).

Methods to extract EPS are important for studying its physicochemical properties and its impact on contaminants in the aquatic environment. Various physical and chemical methods have been reported to extract EPS associated with cells from different sources (e.g. biofilm, sludge and cell suspension). Common physical methods include centrifugation, ultrasonication and heating (Liu and Fang, 2002b; Comte et al., 2007). Chemical methods include extraction of EPS with chemical agents. Ethylenediamine tetraacetic acid (EDTA), formaldehyde, $\mathrm{NaOH}$ and $\mathrm{NaOH}$-formamide are usually used as the extractants (Liu and Fang, 2002b; Adav and Lee, 2008). A review of the literature showed that extraction yield, composition and physicochemical properties of EPS varied greatly with the extraction method. Liu and Fang (2002b) reported that extracting with formaldehyde plus $\mathrm{NaOH}$ obtained more EPS from sludge than other methods including extraction with EDTA, cation exchange resin or formaldehyde. Comte et al. (2007), using high-pressure size exclusion chromatography (HPSEC), demonstrated that extraction of EPS by heating induced hydrolysis of EPS, and extraction with chemical reagents strongly affected the composition of EPS. Mcswain et al. (2005) showed that EPS was contaminated by DNA due to cell lysis during extraction of EPS by heating. The various EPS extraction methods in the literature led to uncertainty and noncomparability of results. Therefore, it is necessary to assess the various extraction methods from the perspective of extraction yield and composition.

Three-dimensional excitation-emission matrix (3DEEM) fluorescence spectroscopy is well known for its speed, good 
selectiveness and high sensitivity in detecting fluorophore substances (Ni et al., 2009). The location of peaks on a 3DEEM fluorescence spectrum provide information on composition of organic matter, and the fluorescence intensity at the characteristic peaks gives information on the relative content of the fluorescent components (Chen et al., 2003; Sheng and Yu, 2006). Three-dimensional excitation-emission matrix fluorescence spectroscopy has been extensively used for studying dissolved organic matter (DOM) (Wu and Tanoue, 2001; Baker, 2002). Recently, 3DEEM fluorescence spectroscopy has also proven to be a useful tool for studying EPS (Sheng and Yu, 2006; Zhang et al., 2009). Limited studies have suggested that there are several peaks in 3DEEM of EPS extracted from activated sludge, being assigned to protein-like substances, humic-like substances and fulvic-like substances (Sheng and Yu, 2006; Adav and Lee, 2008; Ni et al., 2009).

In this study, 5 EPS extraction methods reported in the previous literature were compared in preparing EPS from algabacteria biofilm using 3DEEM fluorescence spectroscopy. The purpose of this study was to assess the pros and cons of these methods and their suitability in preparing EPS samples for scientific research.

\section{Materials and methods}

\section{Samples}

Alga-bacteria biofilm was collected from a pond at the Institute of Geochemistry, Chinese Academy of Sciences, Guiyang, China, using a sterile plastic spatula and placed in a $200 \mathrm{~m} \ell$ autoclaved beaker. Immediately after the biofilm sample was collected, it was washed with de-ionized water and dispersed into suspension by a stirrer. The cell suspension was then immediately subjected to EPS extraction by different methods.

\section{Extraction methods of EPS}

Three chemical methods and 2 physical methods were used to extract EPS from alga-bacteria biofilm. Figure 1 illustrates the processes of these 5 methods. The following 3 chemical methods were assessed for EPS extraction:

- Extraction with EDTA - the cell suspension was extracted with $2 \%$ EDTA for $3 \mathrm{~h}$ at $4^{\circ} \mathrm{C}$.

- Extraction with formaldehyde - the cell suspension was extracted with formaldehyde (36.5\%) for $1 \mathrm{~h}$ at $4{ }^{\circ} \mathrm{C}$.

- Extraction with formaldehyde plus $\mathrm{NaOH}$ - the cell suspension was first extracted with formaldehyde (36.5\%) for $1 \mathrm{~h}$ at $4^{\circ} \mathrm{C}$ and then with $\mathrm{NaOH}\left(1 \mathrm{M}, 4^{\circ} \mathrm{C}, 3 \mathrm{~h}\right.$ ) (Liu and Fang, 2002b).

- All the samples treated with chemical agents were centrifuged at $20000 \mathrm{r} / \mathrm{min}, 4^{\circ} \mathrm{C}$ for $20 \mathrm{~min}$ and the supernatants were filtered through a $0.22 \mu \mathrm{m}$ membrane. The filtrates were used as EPS samples.

The following 2 physical methods were assessed for EPS extraction:

- Extraction with high-speed centrifugation - The cell suspension was centrifuged at $20000 \mathrm{r} / \mathrm{min}, 4^{\circ} \mathrm{C}$ for $20 \mathrm{~min}$. This method was believed not to cause cell lysis (Liu and Fang, 2002b). The supernatants were filtered through a 0.22 $\mu \mathrm{m}$ membrane. The filtrate was used as the EPS sample.

- Extraction with ultrasonication - The cell suspension was firstly subject to ultrasonication at $40 \mathrm{~W}$ in an ice bath for 2 min (Comte et al., 2006). The ultrasound was generated by an ultrasound generator (Sonopuls JY88-II, Ningbo, China). The sonicated cell suspension was then centrifuged at $20000 \mathrm{r} / \mathrm{min}, 4^{\circ} \mathrm{C}$ for $20 \mathrm{~min}$. The supernatants were filtered through a $0.22 \mu \mathrm{m}$ membrane. The filtrate was used as the EPS sample.

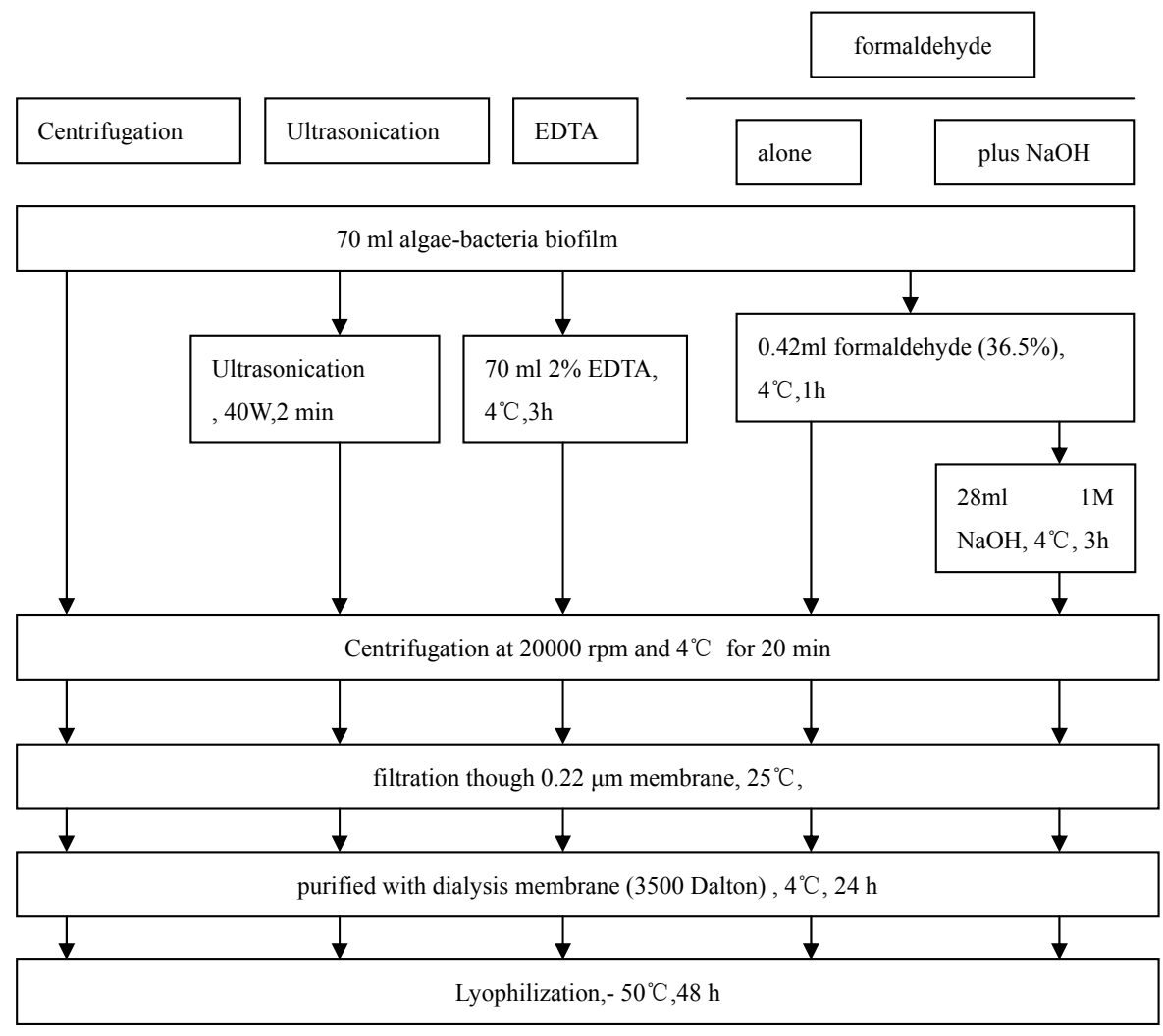

Figure 1

Flow chart of EPS extraction processes 


\begin{tabular}{|l|c|c|c|c|c|}
\hline \multirow{2}{*}{ EPS yield extracted by different methods and its composition before dialysis and after dialysis } \\
\hline Extraction Method & \begin{tabular}{c} 
EPS content \\
before dialysis \\
(mg/g dry \\
\cline { 3 - 6 }
\end{tabular} & \multicolumn{4}{|c|}{ Composition of EPS after dialysis } \\
\cline { 3 - 6 } & & Carbohydrate (mg/g EPS) & \multicolumn{2}{c|}{ Protein (mg/g EPS) } \\
\cline { 3 - 6 } & 7.20 & BD & AD & BD & AD \\
\hline Centrifugation & 12.70 & 56.00 & 38.70 & $--^{* * *}$ & $-^{* * *}$ \\
\hline Ultrasonication & 164.50 & 3.20 & 34.90 & 22.30 & 20.39 \\
\hline EDTA & 14.20 & 25.20 & 1.80 & 1.70 & 0.71 \\
\hline Formaldehyde & 114.70 & 13.30 & 10.42 & 8.00 & 2.57 \\
\hline Formaldehyde- $\mathrm{NaOH}$ & & & & 17.07 & 13.64 \\
\hline
\end{tabular}

$B D^{-}$before dialysis, $A D^{-}$after dialysis, --- undetected

\section{Chemical analysis of EPS}

Polysaccharide content in EPS was determined by the phenolsulphuric acid method, according to Dubois and Gilles (Dubois et al., 1956), with glucose as the standard. The protein content of EPS was determined by the Bradford method (1976) with bovine serum albumin as the standard.

\section{DEEM fluorescence spectroscopy}

All of the EPS samples, before or after dialysis with 3500 Dalton molecular weight cutoff membranes, were characterised with 3DEEM fluorescence by using fluorescence spectrometry (Hitachi, F-4500, Japan). Three-dimensional excitationemission matrix spectra were determined with scanning emission spectra from $250 \mathrm{~nm}$ to $600 \mathrm{~nm}$ at $2 \mathrm{~nm}$ increments by varying the excitation wavelength from $200 \mathrm{~nm}$ to $450 \mathrm{~nm}$ at $5 \mathrm{~nm}$ increments, and with a speed of $1200 \mathrm{~nm} / \mathrm{min}$. The blank spectrum was recorded with double-distilled water. The data were analysed with Sigmaplot 10 (Systat Software Inc., San Jose, USA).

\section{Results and discussion}

\section{Protein and polysaccharide in EPS}

Table 1 summarises the yields and protein and polysaccharide contents of EPS samples prepared with the 5 methods. EPS yield varied significantly with the extraction method. Generally, more EPS was extracted with the chemical methods than with the physical methods. This result was in accordance with some previous studies (Liu and Fang, 2002b; Adav and Lee, 2008 ). The least EPS was extracted with centrifugation. Ultrasonication at $40 \mathrm{~W}$ slightly increased the amount of EPS extracted. EDTA seemed to be most effective in extracting EPS. There are 2 possible reasons for this. One explanation is that EDTA could increase the solubility of EPS and thus increase the yield (Comte et al., 2006). The other explanation is that EPS extracted by EDTA may be overestimated since EDTA may bind with some components in EPS and form complexes that could not be removed by dialysis (Liu and Fang, $2002 b$ ). Extraction with formaldehyde plus $\mathrm{NaOH}$ was also found to be an effective method for EPS extraction. Comte et al. (2006) explained that an increase in $\mathrm{pH}$ due to the presence of $\mathrm{NaOH}$ resulted in separation between acidic groups in EPS and thus more EPS were extracted. In Liu's study (Liu and Fang, 2002b), treatment with formaldehyde plus $\mathrm{NaOH}$ was shown to be the most efficient method.

Composition of EPS was also significantly affected by the extraction method (Table 1). Protein was undetected in EPS samples prepared with centrifugation alone, indicating that little protein was extracted by this method. The protein content was highest in the EPS sample prepared with ultrasonication. The possible reason for this is that ultrasonication may promote the shifts of protein and carbohydrate from the inner layer to the outer layer (Yu et al., 2008). Interestingly, extraction with EDTA gave the highest yield of EPS but the protein and carbohydrate contents were low. This might be explained by the fact that EDTA, carbohydrate and protein form complexes that were not removed by dialysis, resulting in overestimated content of EPS but lower proportions of carbohydrate and protein (Liu and Fang, 2002b).

\section{DEEM fluorescence spectra of EPS}

Four or five peaks were found in each 3DEEM fluorescence spectrum of EPS samples prepared by different methods (Fig. 2). Peak A was at excitation/emission wavelengths (Ex/Em) of 225 to $230 \mathrm{~nm} / 340$ to $372 \mathrm{~nm}$, Peak B at Ex/Em of 275 to $285 \mathrm{~nm} / 346$ to $368 \mathrm{~nm}$, Peak C at Ex/Em of 255 to $270 \mathrm{~nm} / 442$ to $464 \mathrm{~nm}$, Peak D at Ex/Em of 330 to $360 \mathrm{~nm} /$ 390 to $464 \mathrm{~nm}$, and Peak E at Ex/Em of 325to $330 \mathrm{~nm} / 390 \mathrm{~nm}$. Peaks A, B, C and D were found in spectra of all the EPS samples. Peak E was only found in the EPS sample prepared with formaldehyde plus $\mathrm{NaOH}$. Peak A and B were described as protein-like peaks, at which the fluorescence is associated with the aromatic amino acids and tryptophan (Wu and Tanoue, 2001; Baker, 2002; Sheng and Yu, 2006; Adav and Lee, 2008). Fluorescence at Peaks C and D was attributed to the presence of humic acid-like substances (Wu and Tanoue, 2001; Baker, 2002). Peaks A, B, D and E were also reported to be found in the EPS from sludge (Sheng and Yu, 2006; Adav and Lee, 2008; Ni et al., 2009). Peak C was not found in EPS samples in the previous studies. As far as the originality of fluorescence at Peak E was concerned, different studies had different interpretations. Most studies held that fluorescence at Peak E originated from humic acid-like substances (e.g., Wu and Tanoue, 2001; Baker, 2002), but several recent studies demonstrated that it belonged to fulvic acid-like fluorescence (Chen et al., 2003; $\mathrm{Ni}$ et al., 2009).

Location of, and fluorescence intensity at, each peak were clearly affected by the extraction methods (Fig. 2). The location of, and fluorescence intensity at, each peak are listed in Table 2. It was found that fluorescence intensity at Peaks A and B for EPS prepared with centrifugation was much weaker than that for EPS samples prepared with the other 4 methods, indicating that only a small amount of protein-like substances was extracted by centrifugation. This result confirmed the result of undetected protein in the EPS sample prepared with centrifugation (Table 1). Since Coomassie Brilliant Blue has 

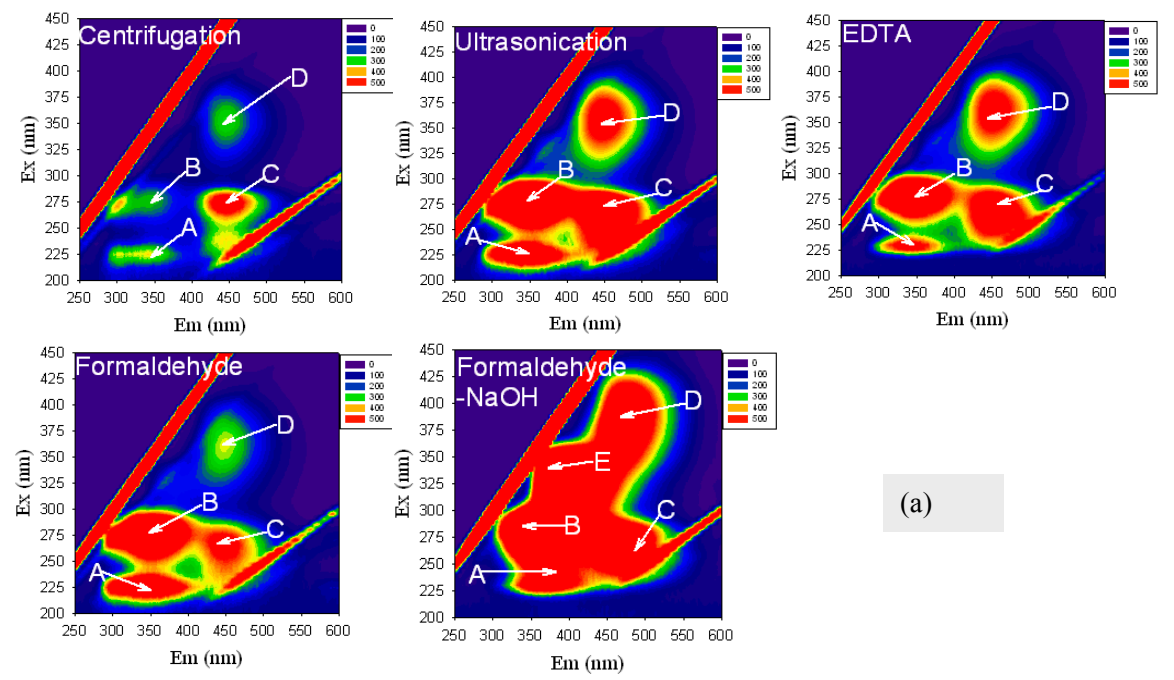

Figure 2

The EEM fluorescence spectra of EPS extracted by using different methods (control, ultrasonication,
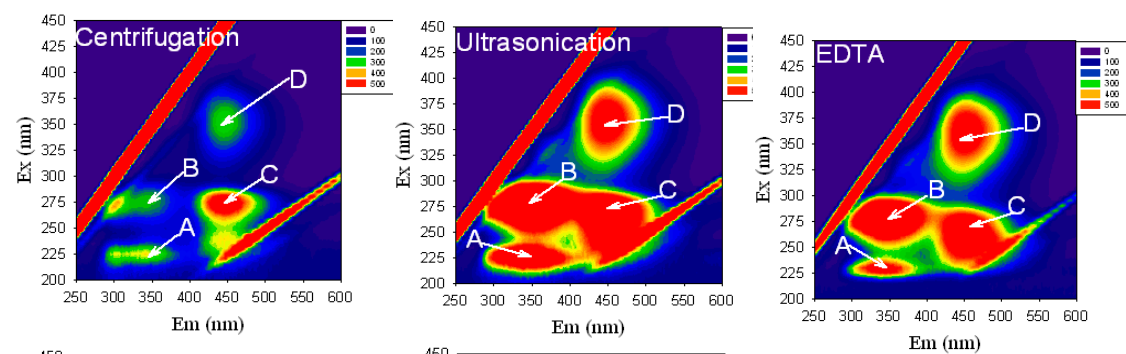

EDTA, formaldehyde and formaldehyde plus $\mathrm{NaOH}$ ),

(a):before dialysis

(b): after dialysis
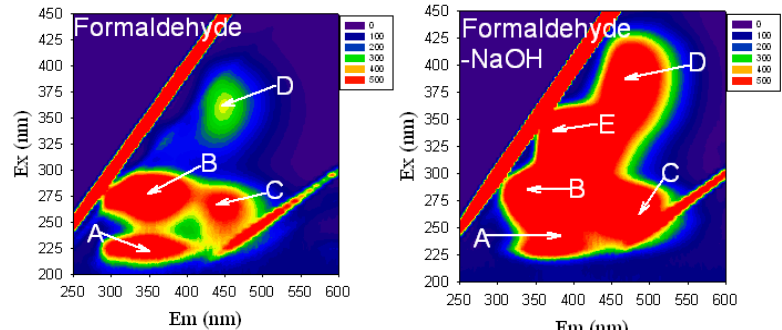

Em (nm)

(b)

\section{Table 2}

Location of and fluorescence intensity at peaks 3DEEM of EPS samples prepared with different methods

\begin{tabular}{|c|c|c|c|c|c|c|}
\hline \multicolumn{2}{|c|}{ Extraction method } & \multirow{2}{*}{$\begin{array}{c}\text { Peak A } \\
\text { Ex/Em intensity }\end{array}$} & \multirow{2}{*}{$\begin{array}{c}\text { Peak B } \\
\text { Ex/Em intensity }\end{array}$} & \multirow{2}{*}{$\begin{array}{c}\text { Peak C } \\
\text { Ex/Em intensity }\end{array}$} & \multirow{2}{*}{$\begin{array}{c}\text { Peak D } \\
\text { Ex/Em intensity }\end{array}$} & \multirow{2}{*}{$\begin{array}{c}\text { Peak E } \\
\text { Ex/Em intensity }\end{array}$} \\
\hline & & & & & & \\
\hline \multirow[t]{2}{*}{ Centrifugation } & $\mathrm{BD}$ & $225 / 346324.7$ & $275 / 352 \quad 438.7$ & $270 / 448921.6$ & $360 / 446 \quad 440.5$ & -- \\
\hline & $\mathrm{AD}$ & $225 / 342 \quad 277.1$ & $275 / 338 \quad 219.1$ & $275 / 448 \quad 669.7$ & $350 / 446 \quad 282.0$ & -- \\
\hline \multirow[t]{2}{*}{ Ultrasonication } & $\mathrm{BD}$ & $225 / 350 \quad 906.4$ & $280 / 350 \quad 1552.0$ & $265 / 450 \quad 1158.0$ & $360 / 448 \quad 660.4$ & -- \\
\hline & $\mathrm{AD}$ & $225 / 336 \quad 1063.0$ & $280 / 340 \quad 1090.0$ & $275 / 446962.2$ & $\begin{array}{ll}350 / 446 & 414.1 \\
\end{array}$ & -- \\
\hline \multirow[t]{2}{*}{ EDTA } & $\mathrm{BD}$ & $230 / 340 \quad 570.3$ & $280 / 346 \quad 192.0$ & $255 / 452 \quad 967.9$ & $360 / 456 \quad 644.5$ & -- \\
\hline & $\mathrm{AD}$ & $230 / 338 \quad 829.7$ & $280 / 334 \quad 1090.0$ & $275 / 446 \quad 606.3$ & $350 / 446 \quad 282.0$ & -- \\
\hline \multirow[t]{2}{*}{ Formaldehyde } & $\mathrm{BD}$ & $225 / 350909.6$ & $275 / 348 \quad 1242.0$ & $265 / 442 \quad 628.0$ & $360 / 448 \quad 326.2$ & -- \\
\hline & $\mathrm{AD}$ & $225 / 338702.7$ & $275 / 340737.0$ & $275 / 442 \quad 513.4$ & $350 / 449284.6$ & -- \\
\hline \multirow{2}{*}{$\begin{array}{l}\text { Formaldehyde } \\
-\mathrm{NaOH}\end{array}$} & $\mathrm{BD}$ & $230 / 372 \quad 775.4$ & $285 / 368 \quad 4381.0$ & $255 / 464 \quad 1195.0$ & $385 / 470 \quad 929.5$ & $330 / 3903067$ \\
\hline & $\mathrm{AD}$ & $230 / 346 \quad 1897.0$ & $280 / 3525432.0$ & $255 / 462 \quad 1675$ & $370 / 464 \quad 890.6$ & $325 / 3901840$ \\
\hline
\end{tabular}

$B D$ - before dialysis; $A D$ - after dialysis; -- - undetected

varying sensitivity to different species of amino acid (Compton and Jones, 1985) and the Bradford assay usually gives lower protein values (Berges et al., 1993), low protein content in the EPS sample could not be detected by the Bradford method. However, 3DEEM fluorescence was found to be a more sensitive method in detecting low content of protein or protein-like substances, which might not be detected by the Bradford method. The weak fluorescence at Peak D for EPS samples prepared with centrifugation alone or formaldehyde alone indicated that a lower proportion of humic-like substances was extracted by these 2 methods compared with the other 3 methods.

Fluorescence intensity was strongest for the EPS sample extracted with formaldehyde- $\mathrm{NaOH}$, indicating that more 
fluorescent components were extracted by this method. The location and shape of peaks for alkali-extracted EPS also differed greatly from the other samples, which might be the result of the high $\mathrm{pH}$ during extraction in the presence of $\mathrm{NaOH}$.

Dialysis was also an important procedure affecting the characteristics of fluorescence spectra of EPS. In most cases, fluorescence intensity at each peak was significantly reduced in the EPS sample dialysed with a 3500 Dalton molecular cutoff dialysis membrane (Table 2). For instance, fluorescence intensity at Peak D in the EPS sample prepared with EDTA decreased by $56 \%$ after dialysis, indicating that a considerable amount of humic acid-like substances with molecular weight of less than 3500 Dalton was removed by dialysis. It was also found that dialysis resulted in a shift of the location of some peaks. Both changes in fluorescence intensity and location indicated that the components with molecular weight of less than 3500 Dalton contained substantive amounts of fluorophores which were lost after dialysis. In some cases, fluorescence intensities were enhanced after dialysis. For example, fluorescence intensities at Peaks A, B and C for $\mathrm{NaOH}$-formaldehyde increased by $144.65 \%, 23.98 \%$ and $40.17 \%$, respectively, after dialysis. This might be explained as follows. Fluorescence of these peaks was quenched due to interaction of the fluorophores with chemicals (e.g., $\mathrm{Na}^{+}$or EDTA) during extraction. Some chemicals bound to EPS were released to water again during dialysis and thus fluorescence intensities of these peaks recovered. Fluorescence of Peak A for EPS extracted with ultrasonication after dialysis also increased a little. This might be explained by the fact that sonolysis led to water dissociation or degradation of some components of EPS and some ions such as $\mathrm{H}^{+}$were produced. These ions might be binding to some fluorophores in EPS during extraction and may be released again during dialysis. Our study suggests that dialysis should be taken into account fully during the preparation of EPS samples.

\section{Conclusions}

The yield of EPS from biofilm varied greatly with extraction method. Pre-treatment with ultrasound of gentle intensity doubled the extraction yield without significant modification of the composition of EPS. Compared with the physical methods, chemical methods significantly increased the extraction yield. However, contamination of the added extractants (e.g. EDTA), interaction of extractants with EPS, and possible release of intracellular components due to the extractant could affect the composition of the EPS considerably. Therefore, appropriate physical methods (for example, ultrasonication at low intensity) for enhancing EPS yields are recommended in research of EPS, and great caution must be taken when chemical methods are used for EPS extraction.

\section{Acknowledgements}

This work was supported by Knowledge Innovation Program of Chinese Academy of Sciences (KZCX2-YW-335 and KZCX2-YW-135), Program of 100 Distinguished Young Scientists of the Chinese Academy of SciencesNational Natural Science Foundation of China $(40673070,40872169)$ and 863 Programme Project 2006AA06Z339 from MOST of China. We are grateful to the editor and the anonymous reviewers for their valuable comments and suggestions.

\section{References}

ADAV SS and LEE DJ (2008) Extraction of extracellular polymeric substances from aerobic granule with compact interior structure. J. Hazard. Mater. 154 (1-3) 1120-1126.

BAKER A (2002) Fluorescence properties of some farm wastes: implications for water quality monitoring. Water Res. 36 (1) 189-195.

BERGES JA, FISHER AE and HARRISON PJ (1993) A comparison of Lowry, Bradford and Smith protein using different protein standards and protein isolated from the marine diatom Thalassiosira pseudonana. Assays Mar. Biol. 115 (2) 187-193.

BHASKAR PV and BHOSLE NB (2006) Bacterial extracellular polymeric substance (EPS): A carrier of heavy metals in the marine food-chain. Environ. Int. 32 (2) 191-198.

BRADFORD MM (1976) A rapid and sensitive method for the quantitation of microgram quantities of protein utilizing the principle of protein-dye binding. Anal. Biochem. 72 248-254.

CHEN W, WESTERHOFF P, LEENHEER JA and BOOKSH K (2003) Fluorescence excitation-emission matrix regional integration to quantify spectra for dissolved organic matter. Environ. Sci. Technol. 37 (24) 5701-5710.

COMPTON SJ and JONES CG (1985) Mechanisms of dye response and interference in the Bradford protein assay. Anal. Biochem. 151 (2) 369-374.

COMTE S, GUIBAUD G and BAUDU M (2006) Relations between extraction protocols for activated sludge extracellular polymeric substances (EPS) and EPS complexation properties Part I. Comparison of the efficiency of eight EPS extraction methods. Enzyme Microb. Technol. 38 (1-2) 237-245.

COMTE S, GUIBAUD G and BAUDU M (2007) Effect of extraction method on EPS from activated sludge: An HPSEC investigation. J. Hazard. Mater. 140 (1-2) 129-127.

DUBOIS M, GILLES KA, HAMILTON JK, REBERS PA and SMITH F (1956) Calorimetric method for determination of sugars and related substances. Anal. Chem. 28 (3) 350-356.

GUIBAUD G, BORDAS F, SAAID A, D'ABZAC P and VAN HULLEBUSCH E (2008) Effect of pH on cadmium and lead binding by extracellular polymeric substances (EPS) extracted from environmental bacterial strains. Colloids Surf., B 63 (1) 48-54.

LASPIDOU CS and RITTMANN BE (2002) A unified theory for extracellular polymeric substances, soluble microbial products, and active and inert biomass. Water Res. 36 (11) 2711-2720.

LIU H and FANG HHP (2002a) Characterization of electrostatic binding sites of extracellular polymers by linear programming analysis of titration data. Biotechnol. Bioeng. 80 (7) 806-811.

LIU H and FANG HHP (2002b) Extraction of extracellular polymeric substances (EPS) of sludges. J. Biotechnol. 95 (3) 249-256.

LIU Y, LAM MC and FANG HHP (2001) Adsorption of heavy metals by EPS of activated sludge. Water Sci. Technol. 43 (6) 59-66.

McSWAIN BS, IRVINE RL, HAUSNER M and WILDERER PA (2005) Composition and distribution of extracellular polymeric substances in aerobic flocs and granular sludge. Appl. Environ. Microbiol. 71 (2) 1051-1057.

MEISEN S, WINGENDER J and TELGHEDER U (2008) Analysis of microbial extracellular polysaccharides in biofilms by HPLC. Part I: development of the analytical method using two complementary stationary phases. Anal. Bioanal. Chem. 39 (3) 993-1002.

NI BJ, FANG F, XIE WM, SUN M, SHENG GP, LI WH and YU HQ (2009) Characterization of extracellular polymeric substances produced by mixed microorganisms in activated sludge with gelpermeating chromatography, excitation-emission matrix fluorescence spectroscopy measurement and kinetic modeling. Water Res. 43 (5) 1350-1358.

SELCK H, DECHO AW and FORBES VE (1999) Effects of chronic metal exposure and sediment organic matter on digestive absorption efficiency of cadmium by the deposit-feeding polychaete Capitella species I. Environ. Toxicol. Chem. 18 (6) 1289-97.

SHENG GP and YU HQ (2006) Characterization of extracellular polymeric substances of aerobic and anaerobic sludge using threedimensional excitation and emission matrix fluorescence spectroscopy. Water Res. 40 (6) 1233-1239. 
SHENG GP, YU HQ and YUE ZB (2005) Production of extracellular polymeric substances from Rhodopseudomonas acidophila, in the presence of toxic substances. Appl. Microbiol. Biotechnol. 69 (2) 216-222.

TSUNEDA S, AIKAWA H, HAYASHI H, YUASA A and HIRATA A (2003) Extracellular polymeric substances responsible for bacterial adhesion onto solid surface. FEMS Microbiol. Lett. 223 (2) 287-292.

WU F and TANOUE E (2001) Isolation and partial characterization of dissolved copper-complexing ligands in streamwaters. Environ. Sci. Technol. 35 (18) 3646-3652.
YU GH, HE PJ, SHAO LM and ZHU YS (2008) Extracellular proteins, polysaccharides and enzymes impact on sludge aerobic digestion after ultrasonic pretreatment. Water Res. 42 (8-9) 1925-1934.

ZHANG DY, WANG JL and PAN XL (2006) Cadmium sorption by EPSs produced by anaerobic sludge under sulfate-reducing conditions. J. Hazard. Mater. 138 (3) 589-593.

ZHANG DY, PAN XL, MOSTAFA KMG, CHEN X, WU FC, SONG WJ and LIU J (2009) Complexation between $\mathrm{Hg}(\mathrm{II})$ and biofilm extracellular polymeric substances: An application of fluorescence spectroscopy. J. Hazard. Mater. 175 (1-3) 359-365. DOI: 10.1016/j. jhazmat.2009.10.011. 